\title{
Equating the brightness of brief visual stimuli of unequal durations
}

\author{
VINCENT DI LOLLO and GARY FINLEY \\ University of Alberta, Edmonton, Alberta, Canada
}

\begin{abstract}
The brightness of brief displays whose durations do not exceed a critical period of integration is known to covary with stimulus duration (Bloch's law). There are cases in which the interdependence of duration and brightness introduces an unwanted confounding that must be avoided. Three methods for equating the brightness of brief stimuli displayed under computer control are described and evaluated, and an example of the outcome obtained with the most efficient procedure is reported.
\end{abstract}

Since the advent of inexpensive microcomputers--just over a decade ago-computer-controlled oscilloscopic displays have been employed with increasing frequency in perceptual and cognitive laboratories. Oscilloscopic displays afford the advantage of excellent resolution in both space and time. Successive stimuli can be displayed in fine spatial alignment with ease unmatched in other sequential display devices, such as tachistoscopes and raster video displays. In the same vein, stimulus duration and succession can be timed with microsecond resolution.

On the debit side, measurement and control of the luminous intensity of oscilloscopic displays is not achieved easily, mainly because the displays are discontinuous both in space and in time. An apparently continuous contour on the face of an oscilloscopic point plotter is actually made up of discrete dots, and the appearance of steadiness is achieved by replotting every dot at a rate higher than the critical fusion frequency (CFF). Sperling (1971) has provided some general guidelines for measuring and reporting the luminous intensity of such displays.

The present paper is concerned not with the luminous intensity of oscilloscopic displays, but with their brightness. In the present paper, luminous intensity is employed as a collective term referring to the amount of light (measured in $\mathrm{cd} / \mathrm{m}^{2}$ ) that is emitted or reflected by an object. The term brightness, on the other hand, refers to the subjective appearance of that object. Although it is reasonable to assume that the subjective sensation of brightness increases with luminous intensity, brightness also varies with other factors that leave luminous intensity unaffected (e.g., size, duration, and retinal location of the stimulus, and state of adaptation of the eye).

More specifically, the present paper is concerned with the problem of equalizing the brightness of brief stimuli displayed for unequal durations. The problem is often en-

This work was supported by Grant A6592 from the Natural Sciences and Engineering Research Council of Canada to the first author. Requests for reprints should be sent to either author at the Department of Psychology, University of Alberta, Edmonton, Alberta, T6G 2E9, Canada. countered when multiple stimuli shorter than a critical duration (typically $100 \mathrm{msec}$ or less) are employed to study such perceptual phenomena as visual masking or temporal integration. It is known that, within the critical duration, the brightness of stimuli displayed at a given luminous intensity per unit of time covaries with exposure duration. It is our aim to examine the procedures that have been employed for equating the brightness of brief displays, to identify any drawbacks, and to suggest improvements. To provide a suitable basis for discussion and to define some fundamental terms, we begin by providing a brief description of the basic structure and functioning of a cathode-ray tube (CRT).

\section{BASIC ASPECTS OF A CRT}

A CRT consists of an evacuated conical glass envelope containing electrical components at its narrow end which produce a tightly focused beam of electrons. This beam is accelerated down the long axis of the tube through an electrical potential change of several kilovolts and strikes the inner surface of the wide end of the cone. The position at which the beam strikes this surface is made visible by a phosphor coating that produces light in the region struck by the beam's energetic electrons. During its passage down the long axis of the CRT tube, the beam can be deflected from the axis by electric fields present between horizontally and vertically opposed pairs of electrically charged plates. Voltages applied across these deflection plates can be used to steer the beam so that it strikes any part of the phosphor-coated display surface at the wide end of the CRT. Electrons have a large chargeto-mass ratio, so a modest amount of energy applied to the deflection plates can produce very high accelerations of the electron beam. This means that the illuminated spot on the display surface can be moved at very high speeds in response to electrical signals, and this makes the CRT display ideally suited to high-speed display applications. A good discussion of the fundamentals of CRT displays can be found in an Application Note published by HewlettPackard Corporation (Bell, 1970). 
In television receivers and computer video-display terminals, the voltages applied to the CRT deflection plates are arranged so that the beam sweeps out a regular pattern of horizontal lines called a raster scan. The standard North American television raster consists of a pattern of 525 horizontal lines that are drawn on the screen 30 times each second. In this type of display, the intensity of the beam is modulated by a signal synchronized with the raster to "paint" an image onto the screen during each scan.

Although raster-type displays have found many uses in the psychological laboratory, their usefulness in vision research has been limited by the fixed rate of plotting. The minimum temporal separation between successive stimuli is $33 \mathrm{msec}$, and the minimum incremental step is also $33 \mathrm{msec}$. These limitations can be avoided by using a point-plotting oscilloscope. In essence, this is a CRT whose beam does not move in raster-scan fashion, but can be moved randomly to any position on the screen. By changing the voltages applied to the deflection plates, the beam can be moved horizontally ( $x$ axis) or vertically ( $y$ axis). In addition, the voltage applied to the beam source ( $z$ axis) can be varied so as to alter the intensity of the dot produced on the display surface.

To illustrate the mode of operation of a point-plotting oscilloscope, imagine that we wish to display two points at two separate locations on the screen. We begin by turning off the $z$ voltage (so as to avoid a visible trace when the beam is moved) and applying the required voltages to the $x$ and $y$ axes. Having thus positioned the notional beam, a point is displayed on the screen by applying a voltage to the $z$ axis for a very brief time (typically $1 \mu \mathrm{sec}$ or less). The intensity of the dot will vary with the magnitude of the $z$ voltage. With the $z$ axis off, the $x$ and $y$ voltages are then adjusted to suit the screen location of the second point, and a brief voltage is again applied to the $z$ axis to intensify the second dot. For displays containing a larger number of dots, this procedure is repeated until all dots have been plotted. To vary the duration of the display, all dots must be reintensified (refreshed) in a continuous cycle to the end of the required temporal interval.

In the present paper, we are concerned exclusively with the brightness equalization of stimuli displayed on oscilloscopic point-plotters, as distinct from raster-scan displays.

\section{STATEMENT OF THE PROBLEM}

The theme of the present paper is best illustrated by an example. In a hypothetical study of backward masking, a test stimulus (TS), such as an alphabetic character, is followed in time by a masking stimulus (MSK), such as an unstructured jumble of contours, that impairs the perception of the TS. The investigator wishes to learn whether the duration of the TS has an effect on the degree of masking. In the hypothetical experiment, duration of MSK is held constant at $50 \mathrm{msec}$, and duration of TS is varied in several steps over a range $10-100 \mathrm{msec}$. If it is found (as is likely) that masking diminishes as duration of TS is increased, the finding cannot be ascribed unambiguously to the effect of duration alone, because this explanation would ignore the duration-intensity reciprocal relationship, commonly known as Bloch's law. On the basis of this relationship (which typically applies to stimuli not exceeding about $100 \mathrm{msec}$ ), the energy of a stimulus is integrated over time so that longer stimuli contain more energy and, hence, appear brighter than shorter stimuli. Therefore, in the hypothetical experiment, the longer stimuli were also brighter and, thus, more visible against the MSK. To unconfound the two factors, we need to vary the duration of the TS without varying its brightness.

Related problems are encountered in studies involving temporal integration of successive brief displays of unequal durations (see, e.g., Di Lollo, 1980; Serviere, Miceli, \& Galifret, 1977). In these studies, the resulting brightness mismatch may prevent temporal integration that might have been possible had all stimuli appeared equally bright.

What is needed is a method for equating the brightness of brief stimuli that are displayed for unequal durations.

\section{A LABORIOUS SOLUTION}

As a first approximation, it may be suggested that the problem can be solved by measuring the luminous intensity of the stimuli through the technique described by Sperling (1971), and then by making the required corrections for stimulus duration according to the reciprocal relationship between duration and intensity. Sperling (1971) suggested that the intensity of a punctate display can be estimated by first measuring the luminance of the display with reference to a known light source and then correcting for rate of refreshing and for number of dots in the display. This yields the measure he calls Luminous Directional Energy (LDE) per point, expressed in candle-microseconds. To return to our problem, suppose that we display a 100 msec stimulus at a refresh rate of $500 \mathrm{~Hz}$ (i.e., each point is refreshed once every $2 \mathrm{msec}$ ); furthermore, by employing Sperling's measurement procedure, we find that the luminous directional energy (LDE) per point is .01 candle- $\mu$ sec. Suppose next that we wish to produce a 50-msec stimulus that appears as bright as the $100-\mathrm{msec}$ stimulus. This can be done entirely on the basis of timeintensity reciprocity without having to change the $\mathrm{LDE}$ per point. All that is needed is to double the rate of refreshing to $1 \mathrm{KHz}$ (i.e., refresh each point every millisecond for a period of $50 \mathrm{msec}$ ).

On the face of it, this procedure should produce two stimuli of equal brightness and different durations. In practice, however, this method has several problems that limit its usefulness. We mention some of them below.

If very brief stimuli (e.g., $1 \mathrm{msec}$ ) are to be displayed, the required rate of refreshing is likely to be very high. In the context of the above example, a 1-msec stimulus would have to be refreshed at a rate of $50 \mathrm{KHz}$ to appear 
as bright as the 100 -msec stimulus. This would require that each point be refreshed once every $20 \mu \mathrm{sec}$. Although this is technically quite feasible (current random-plotting devices are capable of 2-MHz rates; see Finley, 1985), the high frequency of replotting may run afoul of the decay time of even very fast phosphors, such as P15. Under these conditions, the next intensification would arrive at a time when the phosphor has not yet recovered from the effects of the previous intensification. This would introduce a nonlinearity that could result in stimuli containing significantly less energy than would be expected on the basis of the higher plotting rate.

A second limitation is, in some sense, the converse of that described above. If the initial display contains a large number of points (as would be required to represent a face or a scene), then an upper limit to the plotting rate would be reached very quickly even with a fast plotting device. An option, in this case, would be to increase the brightness of the briefer stimulus, not by employing a faster rate of plotting but by increasing the $z$ voltage of the oscilloscope. This would increase the number of electrons directed at the screen at each intensification and would produce brighter dots. In terms of the above example, it would be possible to produce a 50 -msec stimulus that appeared as bright as a 100 -msec stimulus by leaving the refresh rate unaltered at $500 \mathrm{~Hz}$ and increasing the $z$ voltage to produce a level of LDE double that of the 100 msec stimulus, namely .02 candle- $\mu$ sec. Of course, a combination of changes in plotting rate and $z$ voltage could be used.

A complication of this approach is that the luminous output of the phosphor in commercially available oscilloscopes is not related linearly, or even simply, to $z-$ voltage levels, although the relationship is monotonic. Thus, a doubling of the LDE value cannot be achieved by simply doubling the $z$ voltage. What is required is a prior calibration in which a photometer and Sperling's procedure are employed to measure the luminous output of the oscilloscope at each $z$-voltage level. A table of correspondences (a look-up table) can thus be constructed from which to read the specific $z$ value required to obtain the desired increment in LDE.

Yet a third, and most telling, problem besets this method. It has to do with the length of the critical period of integration over which duration-intensity reciprocity can be expected to occur. This critical period was first reported by Bloch (1885, cited in Uttal, 1981) for stimuli near absolute threshold, but has been reported also for stimuli well above threshold (e.g., Butler, 1975). However, even though the reciprocal relationship holds both at threshold and above, the duration of the critical period is known to decrease dramatically as the stimulus rises above threshold. Indeed, the duration of the critical period varies significantly with other factors, such as dark adaptation and conditions of viewing. An informative review of this topic has been recently provided by Uttal (1981, p. 528 ff).
With reference to the method under discussion, this means that, in order to carry out the brightnessequalization procedure, it is first necessary to find out the duration of the critical period pertaining to the specific experimental conditions in which the brightness-equalized stimuli are to be employed. This can be a time-consuming undertaking.

By now it must be quite obvious that this method of equating the brightness of brief stimuli is long, laborious, and probably prone to error. Even small rounding errors made at the initial stage of estimating LDE per dot per unit time (the Sperling measurement procedure) can develop into a noticeable brightness difference once the rest of the computations have been carried out. We have chosen to comment on this method in some detail, despite its awkwardness, in order to highlight the drawbacks of what might appear, at first, to be a simple and straightforward procedure.

\section{A SIMPLER SOLUTION}

A brightness-equalization procedure that requires neither a measurement of luminous intensity nor prior knowledge of the length of the critical period of integration was described independently by Di Lollo (1977, 1979) and by Serviere et al. (1977). It is an entirely pragmatic procedure in which an observer is simply required to adjust the luminous intensity of one of two simultaneously displayed stimuli (comparison stimulus, COMP) until it appears of the same brightness as the other stimulus (standard stimulus, STD).

For example, suppose that an experiment requires five stimuli of equal brightness but of unequal duration (e.g., $20,40,60,80$, and $100 \mathrm{msec}$ ). In order to appear equally bright, the five stimuli must be plotted at five different $z$ levels whose values are to be established in a preliminary calibration run. For simplicity, assume that each stimulus consists of a vertical rectangular grid of 3 (horizontal) $\times 5$ (vertical) closely-spaced dots. We begin by selecting one stimulus as the STD; typically, this will be the longest stimulus. Next, we select one COMP stimulus from the remaining four, and we display STD and COMP side by side on the display surface. The control program is written so that the $z$ value of STD remains constant, but that of COMP is under the control of the observer who is required to adjust it until its brightness matches that of STD. The procedure is repeated with each COMP so as to yield five separate $z$ values to be used in the experiment proper.

Although the procedure is extremely simple, the outcome can be totally invalidated unless some precautions are observed. First, STD and COMP must be temporally aligned so as to obviate suppression through metacontrast or apparent motion. For example, consider an $80-\mathrm{msec}$ STD and a 40-msec COMP: if their onsets are simultaneous, STD will outlast COMP by $40 \mathrm{msec}$. This display has two compelling aspects: there is a strong appearance 
of motion toward STD, and COMP is noticeably dimmer than when displayed alone. On the other hand, if STD and COMP are made to terminate together, the direction of apparent motion is toward COMP, and now it is STD that appears dimmer than when displayed alone. These are both instances of metacontrast suppression (e.g., Kahneman, 1967). In either case, the $z$ value ultimately assigned to COMP is inappropriate: it will be either an overestimate (in the former case) or an underestimate of the appropriate value.

Fortunately, a simple rule of thumb permits us to steer clear of both Scylla and Charybdis. We have found that neither apparent motion nor suppression is obtained if COMP is displayed halfway through the duration of STD. In terms of the above example, the display would start with a 20-msec exposure of STD alone, would continue with STD and COMP for $40 \mathrm{msec}$, and would end with STD alone for $20 \mathrm{msec}$. Why apparent motion and suppression should not occur under these display conditions is an interesting question to which there is no firm answer at present. Nevertheless, this rule of thumb must be followed if brightness equalization is to be achieved with this method.

Next, the refreshing rate for the stimuli in the experiment proper must be the same as in the preliminary run. If the rates differ, the total amount of energy falling within the critical period of integration will vary, and so will stimulus brightness.

Finally, the viewing conditions in the preliminary run should match closely those of the experiment proper. Notably, if the state of adaptation of the eye differs in the two situations, the duration of the critical period of integration will also differ, with dire consequences for brightness equalization.

It should be noted that, as well as being simple to implement, this technique has no requirements for linearity with respect to any part of the system, including the relationship between $z$ voltage and luminous output. The usefulness of the method has been confirmed both in studies of masking (e.g., Di Lollo, 1980) and in studies of temporal integration (e.g., Serviere et al., 1977).

\section{AN IMPROVED SOLUTION}

To perform the brightness-equalization task described above, the observer must develop a suitable criterion on which to base a decision of whether or not STD and COMP appear to have the same brightness. This might create some concerns regarding the stability of the criterion across individuals, and the possibility of response-criterion bias. These concerns can be alleviated by employing a criterion-free method such as the twoalternative forced-choice (2AFC) method.

Implementation of the 2AFC method is relatively simple: the screen locations (left or right) of STD and COMP are set randomly on each trial, and the observer is required to say which stimulus (left or right) is brighter. The control program keeps track of the screen location of the stimuli on each trial and adjusts the luminous intensity of COMP in accordance with the observer's responses. The aim is to converge to a luminance of COMP at which the observer names STD and COMP equally often as being the brighter stimulus (i.e., a level at which the observer can no longer discriminate between the two stimuli in terms of brightness). Convergence to such a level is achieved most conveniently and rapidly by employing a dynamic threshold-tracking technique, such as PEST (Taylor \& Creelman, 1967).

In its regular usage, PEST is employed to estimate stimulus values that yield a predetermined level of performance. For example, suppose that we wish to find the $75 \%$ increment-threshold of luminance between two otherwise identical stimuli (STD and COMP): that is, we wish to find the level of luminance at which COMP must be displayed in order to be judged as being the brighter stimulus $75 \%$ of the time. The observer is required to indicate which of the two simultaneously displayed stimuli is brighter. If the observer makes too many correct responses, PEST lowers the luminance of COMP; if too many errors are made, the luminance of COMP is increased. This continues until a level of $75 \%$ correct responses is achieved, as indicated by the outcome of a Wald (1947) sequential likelihood-ratio test.

This procedure can be adapted readily to the purpose of brightness equalization: given a brief COMP and a longer STD, we wish to find the level of luminance at which COMP must be displayed in order to be judged as the brighter of the two stimuli $50 \%$ of the time. A response can be arbitrarily defined as "correct" when COMP is judged as being brighter, and as "incorrect" when STD is judged as brighter. When the $50 \%$ level is reached, PEST returns with the level of luminous intensity at which COMP must be displayed in order to be indistinguishable from STD in terms of brightness.

Obviously, the $50 \%$ level could also be reached, and very rapidly, if the observer's eyes were shut and the responses were random. Therefore, this procedure requires a modicum of cooperation from the observer. Otherwise, the method yields remarkably fast and stable estimates, as shown in Figure 1. The curve labeled "equal brightness" was obtained by employing PEST to find the $z$ values at which stimuli shorter than 100 msec must be plotted in order to appear as bright as a $100 \mathrm{msec}$ stimulus displayed at a $z$ value that made it comfortably visible. The oscilloscope was a Hewlett-Packard 1333A that was equipped with $\mathrm{P} 15$ phosphor and was controlled by a PDP-11/34A computer.

Are the results pictured in the "equal brightness" curve in accordance with a reciprocal relationship between duration and intensity (Bloch's law)? An answer to this question cannot be provided solely on the basis of the data in the "equal brightness" curve because, as mentioned above, the relationship between $z$ levels and the corresponding levels of luminous intensity is not known. What is needed is an estimate of the luminous intensity produced by each $z$ value. This estimation was reached 


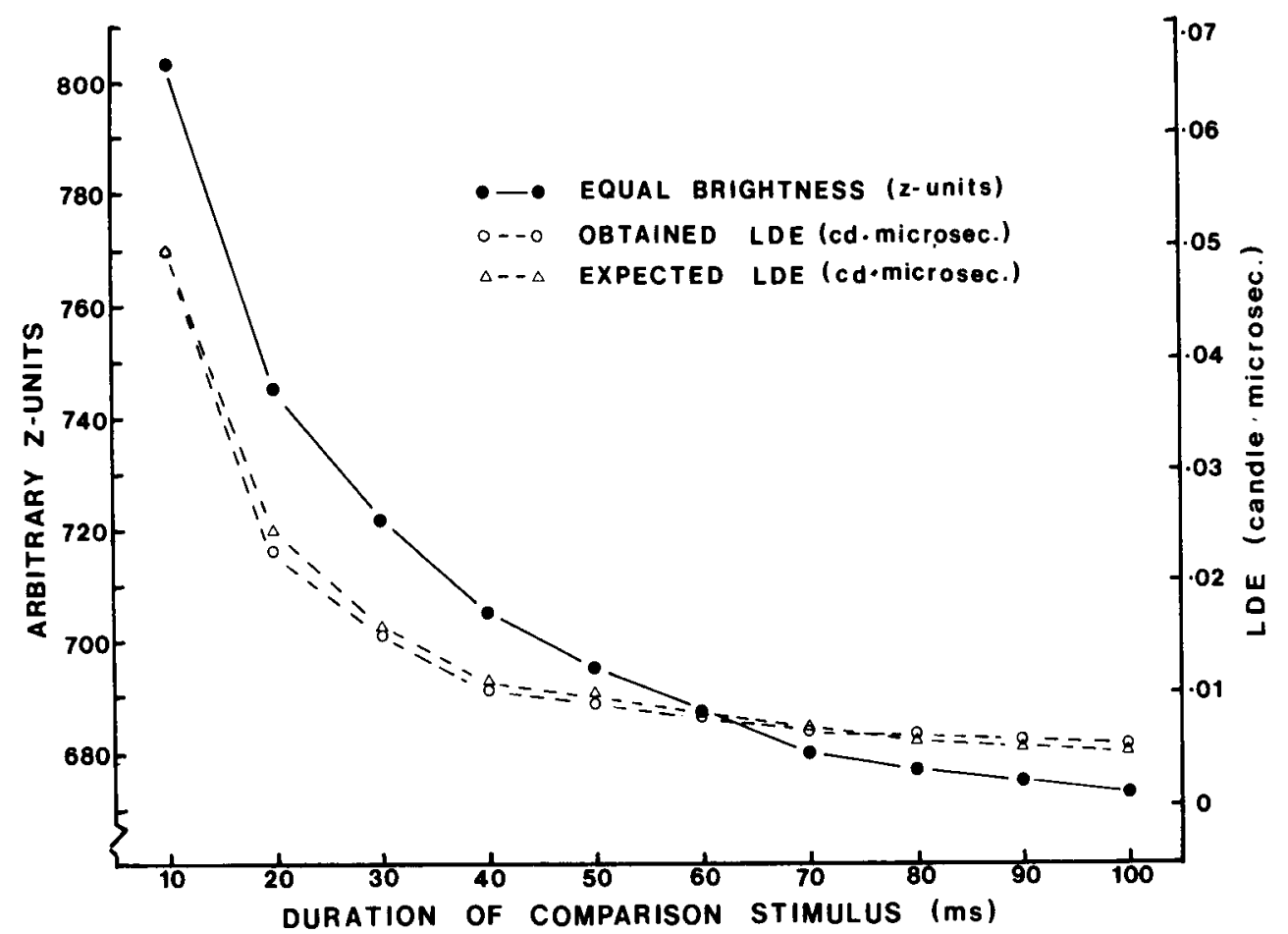

Figure 1. Continuous line: outcome of the brightness-equalization procedure. Segmented lines: values of luminous directional energy (LDE) per point obtained through direct measurement (open circles), and expected on the basis of time-intensity reciprocity (triangles). See text for explanation.

by employing Sperling's (1971) method to measure the LDE corresponding to each relevant $z$ value.

A square patch consisting of a grid of $100(10 \times 10)$ closely spaced dots was cycled continuously on the display surface, at the $z$ level corresponding to a stimulus duration of $10 \mathrm{msec}$ in the "equal brightness" curve (Figure 1). The luminance of the patch was then measured by means of a Spectra Spot Meter, and the reading was transformed into LDE per point according to Sperling's (1971) prescription. Next, the $z$ level of the patch was changed to that corresponding to a stimulus duration of $20 \mathrm{msec}$ in the "equal brightness" curve, and a second measurement was taken. This procedure was repeated for the remaining eight points in the "equal brightness" curve. The 10 readings expressed in LDE values are shown in the curve labeled "obtained LDE" in Figure 1. The companion curve, labeled "expected LDE," shows the LDE values that would be expected on the basis of a simple relationship between intensity and duration. The correspondence between the two LDE curves is quite close, testifying to the stability of the brightness-equalization procedure, and suggesting that, under the viewing conditions employed in the test, the duration of the critical period of integration was at least $100 \mathrm{msec}$.

\section{REFERENCES}

BELL, R. A. (1970). Principles of cathode-ray tubes, phosphors, and high-speed oscillography (Application Note 115). Colorado Springs, CO: Hewlett-Packard Corp., Oscilloscope Systems Division.

ButLER, T. W. (1975). Luminance-duration relationships in the photopic ERG and the apparent brightness of flashes. Vision Research, 15, 693-698.

Di Lol.to, V. (1977). Temporal characteristics of iconic memory. $\mathrm{Na}$ ture, 267, 241-243.

Di LoLlo, V. (1979). Luminous calibration of oscilloscopic displays. Behavior Research Methods \& Instrumentation, 11, 419-421.

Di LoLlo, V. (1980). Temporal integration in visual memory. Journal of Experimental Psychology: General, 109, 75-97.

Finley, G. (1985). A high-speed point plotter for vision research. $V_{i}$ sion Research, 25, 1993-1997.

KaHneman, D. (1967). An onset-onset law for one case of apparent motion and metacontrast. Perception \& Psychophysics, 2, 577-584.

Serviere, J., Miceli, D., \& Galifret, Y. (1977). A psychophysical study of the visual perception of "instantaneous" and "durable." $V i$ sion Research, 17, 57-63.

SperLing, G. (1971). The description and luminous calibration of cathode ray oscilloscope visual displays. Behavior Research Methods \& Instrumentation, 3, 148-151.

Taylor, M. M., \& Creelman, C. D. (1967). PEST· Efficiency estimates on probability functions. Journal of the Acoustical Society of America, 41, 782-787.

UtTAL, W. R. (1981). A taxonomy of visual processes. Hillsdale, NJ. Erlbaum.

WALD, A (1947). Sequential analysis. New York: Wiley 